\title{
The role of peripheral vision in implicit contextual cuing
}

\author{
Marieke van Asselen and Miguel Castelo-Branco \\ University of Coimbra, Coimbra, Portugal
}

\begin{abstract}
Implicit contextual cuing refers to the ability to learn the association between contextual information of our environment and a specific target, which can be used to guide attention during visual search. It was recently suggested that the storage of a snapshot image of the local context of a target underlies implicit contextual cuing. To make such a snapshot, it is necessary to use peripheral vision. In order to test whether peripheral vision can underlie implicit contextual cuing, we used a covert visual search task, in which participants were required to indicate the orientation of a target stimulus while foveating a fixation cross. The response times were shorter when the configuration of the stimuli was repeated than when the configuration was new. Importantly, this effect was still found after 10 days, indicating that peripherally perceived spatial context information can be stored in memory for long periods of time. These results indicate that peripheral vision can be used to make a snapshot of the local context of a target.
\end{abstract}

Our environment contains a large amount of visual information, such as different objects, buildings, and faces, making it impossible to process this complex information at once. In order to compensate for the limitations of the visual system, attention is allocated to the most relevant visual information. Attention can be guided by bottom-up and top-down processes. That is, a specific visual feature can attract attention (e.g., a fast-moving object or a bright color), resulting in an eye movement to the source of information (bottom-up process). In addition, knowledge about our environment can guide attention (top-down process).

One of the top-down mechanisms that guide attention is a result of implicit contextual learning, as was initially shown by Chun and Jiang (1998). They suggested that visual information from our environment can be learned implicitly and can subsequently guide attention to a specific target location. That is, the association between a target and its surrounding visual context (such as spatial information) can be memorized, improving performance on a visual search task. The contextual-cuing paradigm is typically used to study implicit contextual learning of spatial information. It involves a visual search task, in which a rotated target stimulus (T) is presented among a number of rotated distractors (Ls). The participants have to locate the target as quickly as possible and indicate the direction of rotation. Half of the spatial configurations (i.e., positions of the stimuli) are repeated during the experiment. Interestingly, response times are shorter when the configurations are repeated than when they are new, indicating that contextual information was memorized (Chun, 2000; Chun \& Jiang, 1998; Peterson \& Kramer, 2001). Participants are not aware of the repetitions and perform at chance level on a recognition memory task, indicating that it is an implicit memory process. This effect is found after a few repetitions (Chun \& Jiang, 1998) and remains for weeks after testing (Chun \& Jiang, 2003; Jiang, Song, \& Rigas, 2005), indicating that it is a robust mechanism.

Recently, Brady and Chun (2007) proposed a model of implicit contextual learning, based on the idea that contextual learning results from the pairwise statistical association between the distractor locations and the target. They used this model to predict the outcome of different contextual-cuing tasks. An important and interesting aspect of the model is that it includes a spatial constraint, assuming that learning is restricted to the local area around the target. Thus, a very limited amount of contextual information is learned, which is spatially close to the target. They stated that "observers may be encoding just one snapshot of the local context surrounding the target when it is detected" (p. 813). Brady and Chun tested this model by comparing modeling results with behavioral results under different task conditions and found that the model was accurate in predicting the behavioral results of various experimental studies. The idea that implicit contextual learning is restricted to local context information was based on the results of Olson and Chun (2002). In a series of experiments, they manipulated the context by repeating spatial information in half of the screen. Thus, contextual information was repeated on the same side of the target or on the opposite side of the target. It was found that the contextual-learning effect was found in the short-range predictive condition but not in the long-range predictive condition. These behavioral results were later compared

M. van Asselen, masselen@ibili.uc.pt 
with the modeling results of Brady and Chun and were found to be comparable (Brady \& Chun, 2007).

The spatial constraint that is part of the contextuallearning model of Brady and Chun (2007) raises the question of how such a "snapshot" of the local context is made. We suggest that peripheral vision has an important role in making such a snapshot. When locating a target, we tend to keep our eyes focused on the target. This implies that to learn the contextual information, we have to use peripheral vision. Our visual system is divided into central and peripheral vision. Central vision refers to information that is sensed by the central retina - that is, the foveomacular region (which is the part of the eye with which one fixates). It processes high-resolution details, although it covers only a small part of the visual world (circa $1^{\circ}$ of visual angle). In contrast, peripheral vision covers a much larger area of the visual world but processes only low spatial frequencies and degraded color information. In real life, we perceive a large amount of visual information without actually making eye movements toward the source of information, thus using peripheral vision. For example, when we are walking to a place that we can see from afar, we might focus our eyes on that place already as we are walking to it. In the meantime, we still perceive information from the peripheral visual field. It seems unlikely that as we arrive at our destination, we would have no memory of the environment we had walked through.

In order to define whether peripheral vision can be used to make a snapshot of the local context around a target during implicit contextual learning, we used a visual search task in which subjects had to fixate a cross in the middle of the computer screen. The stimuli were presented around the fixation cross, and the subjects were instructed to covertly locate a target stimulus as quickly as possible and indicate the direction of its orientation without taking their eyes off the fixation cross. Importantly, the visual search task included repeated and new configurations. Eye movements were recorded to define whether the subjects were foveating the fixation cross and not the stimuli. A recognition memory task was included at the end of the experiment in order to verify whether the nature of learning was truly implicit or whether the subjects were, to some extent, aware of the repetitions and had explicitly learned some of the information. In an additional experiment, we tested whether context information that is learned with peripheral vision remains in memory for a long time, as had been found in previous studies in which free viewing was used (Chun \& Jiang, 2003). This second experiment, which took place around 10 days after the first experiment, included the configurations that were used in the first experiment.

\section{METHOD}

\section{Subjects}

Thirty-three healthy young subjects participated in this study. Seventeen of these subjects were excluded from further analyses because they were unable to maintain fixation during the entire experiment or due to problems with the eyetracking (head movements during recording, subjects with contact lenses, problems with calibration). The remaining 16 subjects who were included had a mean age of
24.1 years and 16.3 years of education on average. They were all right-handed and had normal or corrected-to-normal visual acuity.

\section{Apparatus and Stimuli}

The stimuli were programmed in Presentation (Neurobehavioral Systems). Six stimuli were shown on each trial, including five distractors ( $(\llcorner)$ and one target ( $\mathrm{T}$ ) (see Figure 1). The distractor stimuli were rotated $0^{\circ}, 90^{\circ}, 180^{\circ}$, or $270^{\circ}$. The target stimulus was rotated $90^{\circ}$ or $270^{\circ}$. The background color was light gray and the stimuli were black. The luminance of the background was $132.9 \mathrm{~cd} / \mathrm{m}^{2}$, whereas the luminance of the stimuli was $0.4 \mathrm{~cd} / \mathrm{m}^{2}$. The stimuli were $0.81^{\circ} \times 0.81^{\circ}$. The distance between the computer screen and the subjects' eyes was $52 \mathrm{~cm}$. The stimuli were presented in an area starting at a distance of $2.4^{\circ}-4.1^{\circ}$ around the fixation cross, meaning that the closest stimulus could be presented $2.4^{\circ}$ from the fixation cross and that stimuli could be presented as far as $4.1^{\circ}$ away. Thus, the subjects could never fixate a stimulus. In order to control whether the subjects fixated the fixation cross, eye movements were measured with the iViewX High-Speed Eye-Tracker (SMI). The fixation cross had a dimension of $0.65^{\circ} \times 0.65^{\circ}$. Trials on which the subjects did not fixate within $1^{\circ}$ of visual angle of the cross were discarded. A fixation was defined as occurring when visual gaze was maintained for $150 \mathrm{msec}$.

\section{Procedure}

The subjects were instructed to look at a fixation cross during the whole experiment and to locate the target stimulus as quickly as possible, without moving their eyes from the fixation cross. Subsequently, they had to indicate the direction of rotation of the target stimulus and press one of two buttons on the keyboard. The experiment consisted of 24 blocks of 12 trials, resulting in a total of 288 trials. Each block included six configurations that were repeated during the experiment (once per block) and six new configurations. In the trials with a repeated configuration, the positions of the distractors and target " $\mathrm{T}$ " were constant; however, the direction of rotation of the target "T" was randomly defined. During the experiment, two short $(10$-sec) intervals were given in which the subjects were allowed to move their eyes freely. The experiment started with 24 practice trials, which were repeated if the subjects had difficulty in understanding the task or fixating the cross.

After the contextual-cuing task, the subjects were asked three questions: (1) Did you notice anything during the experiment? (2) Did you notice that some of the configurations were repeated? and (3) Did you try to remember the repeated configurations? Subsequently, a recognition task was performed in which the six repeated configurations were randomly shown among six new configurations. The subjects were asked to indicate whether they recognized the configuration or not. No eye movements were recorded in this control task, nor were the subjects instructed to look at the fixation cross.

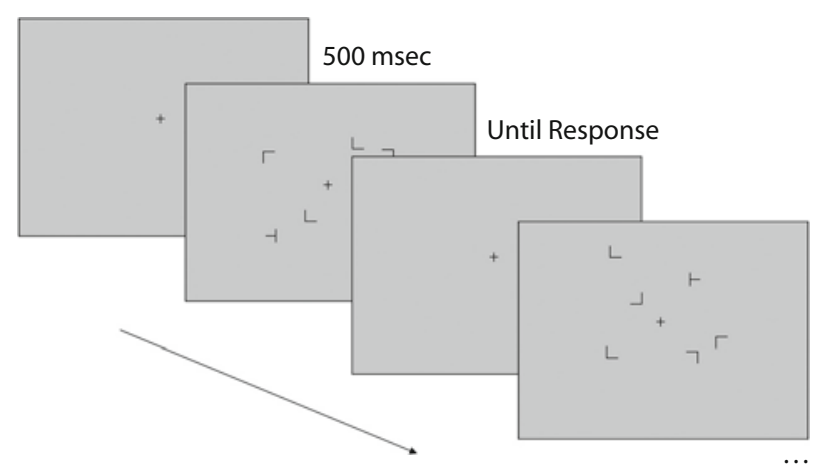

Figure 1. Example of a few displays for the contextual-cuing task. 
To determine whether implicit contextual learning remains in memory during an extended time period, the subjects performed a second contextual-cuing task including the same repeated configurations about 10 days after participating in the first experiment. Due to circumstances, not all the subjects could be tested after exactly 10 days, resulting in an average delay of 11 days. The experiment consisted of eight blocks of 12 trials. Each block included the six configurations that were used in the first experiment (repeated-old) and six new configurations that were repeated during the second experiment (repeated-new). This repeated-new condition was included in order to control for new learning during the second session. If the repeated-old configuration had been compared with new-new configurations, it would not have been possible to distinguish between the two following possibilities: (1) The effect is due to persistence in long-term memory, and (2) the effect is due to the learning that occurs within a session. Since Effect 2 had already been documented in Experiment 1, the critical question in Experiment 2 was whether the learning effect would persist, and a new-new configuration would have been uninformative in this respect. After this contextual-cuing task, the same three questions as those in the first experiment were asked, after which a recognition memory task was performed. The latter task included the six repeated-old and six repeated-new configurations that were used during the contextual-cuing task, as well as six new configurations. All other aspects were the same as those in Experiment 1.

\section{RESULTS}

\section{Experiment 1}

Eye movements. The subjects were able to maintain fixation on $82.1 \%$ of the trials. The trials in which fixation was not maintained were excluded from further statistical analyses. Mean distance from fixation (i.e., the distance between a fixation and the fixation cross) for the trials on which fixation was maintained was $0.5^{\circ}$ of visual angle. For the trials on which the subjects did not maintain fixation, the average distance from fixation was $1.2^{\circ}$ of visual angle.

Contextual-cuing task. Mean reaction times in the first experiment are shown in Figure 2. For statistical analyses, the 24 blocks were averaged into six epochs. The subjects made very few errors (mean, 2.9\%). Trials were excluded on which an incorrect response was given, or that had a response time of more than two $S D$ s above the mean response time of each individual subject (mean, 5.1\%).

A $2 \times 6$ repeated measures ANOVA was conducted, with within-subjects factors of epoch (1-6) and condition (repeated and new trials). A main effect for condition $[F(1,15)=14.8, p<.01]$ was found, indicating that reaction times were significantly shorter in the repeated condition than in the new condition. Reaction times decreased during the session, as is reflected in the main effect for epoch $[F(5,75)=9.0, p<.01]$. An interaction effect was found for block $\times$ session $[F(5,75)=2.3, p=$ $.05]$. When separate paired-sample $t$ tests were performed, a significant effect was found for the last epoch $[t(15)=$ $3.1, p<.01]$, but not for the first epoch $[t(15)=1.6]$. This interaction effect demonstrates that the difference in response times between the repeated and the new trials is larger at the end of the experiment (i.e., when learning has taken place), when compared with the beginning of the experiment, thus reflecting contextual learning during the experiment.
Since some subjects made an erratic saccade outside the fixation area on a few of the trials, it could be argued that implicit contextual learning has taken place during these saccades. In this case, the subjects who made more saccades outside the fixation area should show a larger contextual-cuing effect than the subjects who made no or very few saccades outside the fixation area. Therefore, we have analyzed the relation between the percentage of saccades outside the fixation area and the extent of contextual learning (difference in reaction times between the new and old for Epochs 1-6). A one-tailed correlation analysis indicated no significant effect $(r=-.1, p=.7)$, thus ruling out the possibility that the few saccades that were made outside the fixation area were responsible for the contextual-cuing effect.

Recognition memory task. In response to the first question, none of the subjects reported having noticed the repeated configurations. In response to the second question, 1 subject reported having noticed one repeated configuration, and 2 other subjects reported having noticed a few configurations. However, none of the subjects tried to remember the configurations. Mean correct responses for the repeated and new trials were 3.3 and 3.1, respectively. A one-sample $t$ test indicated no significant difference between correct responses on the repeated $[t(15)=0.8]$ and new $[t(15)=0.2]$ trials, in comparison with chance level (3 correct).

\section{Experiment 2}

Eye movement data. Eight subjects were tested again 10 days after the first contextual-cuing task. During this second contextual-cuing task, fixation was maintained on $79.2 \%$ of the trials. Trials on which no fixation was maintained were excluded from further statistical analyses.

Contextual-cuing task. Mean reaction times for the old and new trials as a function of epoch (1 or 2) are shown in Figure 3. For statistical analyses, the eight blocks were averaged into two epochs. Trials on which an error was

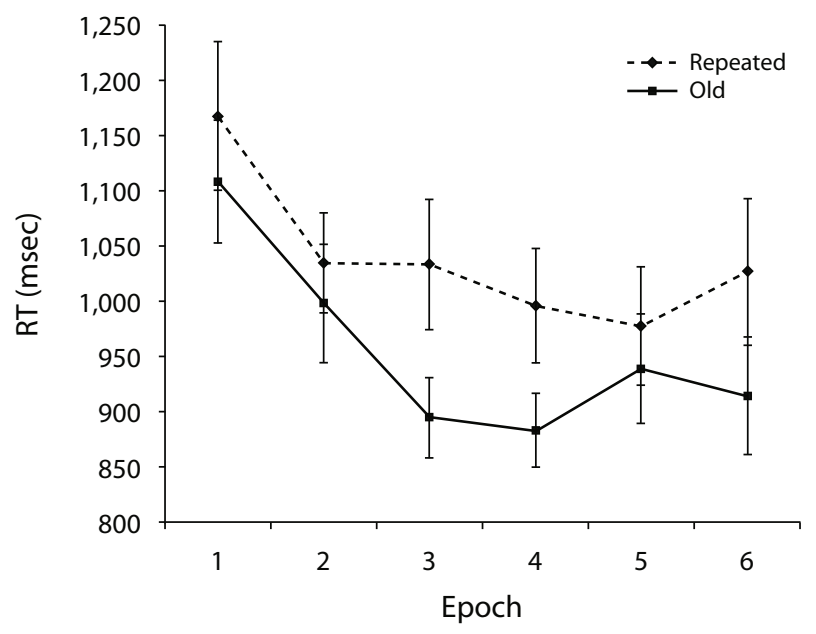

Figure 2. Mean reaction times (RTs) and standard errors for the repeated and old trials in the first session as a function of epoch (1-6). 


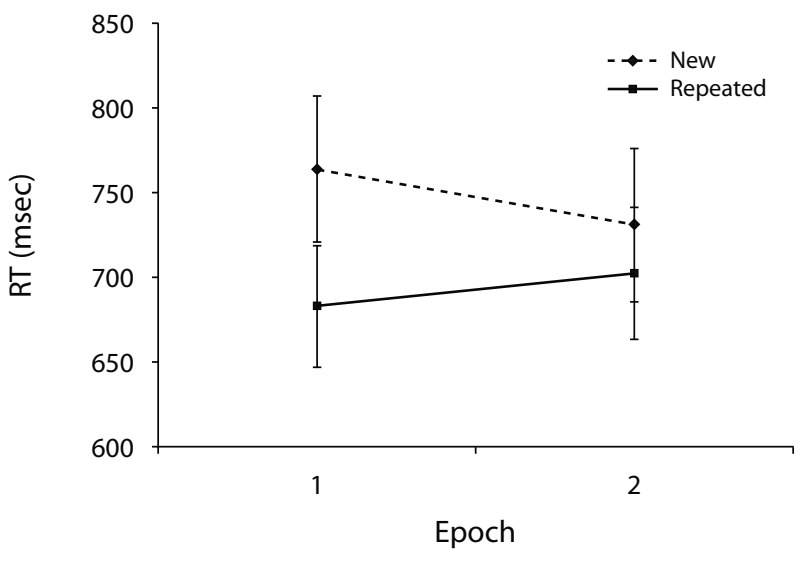

Figure 3. Mean reaction times (RTs) and standard errors for the new and repeated trials in the delayed recall task as a function of epoch (1 or 2).

made $(2.5 \%$ on average) were excluded from analyses, as well as trials on which the response time was longer than two $S D$ s above the average for each individual separately $(5.2 \%$, on average). The data were then subjected to a $2 \times 2$ repeated measures ANOVA, with within-subjects factors of epoch ( 1 or 2 ) and condition (repeated-old or repeated-new trials). A main effect was found for the condition variable $[F(1,7)=7.2, p<.05]$, indicating shorter response times on the repeated-old trials. No main effect was found for epoch $[F(1,7)=0.0]$. However, an interaction effect was found between condition and epoch $[F(1,7)=10.9, p<.05]$, indicating that the difference between the old and new trials was larger in the first epoch than in the second (as would be expected from the fact that repeated-new configurations remain new only during the first epoch).

Recognition memory task. None of the subjects reported having noticed the repetitions, nor did they try to memorize the configurations. Mean correct responses were calculated for the repeated-old (2.9), repeated-new (2.5), and new trials (3.3). One-sample $t$ tests indicated that there was no significant difference between chance level ( 3 correct responses) and correct responses for the repeated-old $[t(7)=0.8]$, repeated-new $[t(7)=0.2]$, and new $[t(7)=0.7]$ trials.

\section{DISCUSSION}

The aim of the present study was to define whether implicit contextual learning can take place in peripheral vision. Therefore, a visual search task was designed in which subjects had to foveate a fixation cross while covertly searching for a target stimulus among a number of distractors that were presented in the peripheral visual field. The results of the first experiment show that when configurations were repeated, response times were shorter, indicating that contextual information was memorized. The subjects did not notice the repetitions, nor were they able to identify repeated configurations on a subsequent recognition memory task, underlining the implicit nature of this memory process. These findings provide evidence for the idea that detailed foveal perception is not necessary for the contextual-cuing effect to occur. This is particularly interesting, since recent studies have shown that it is the contextual information in spatial proximity to the target that is important for guiding attention (Brady \& Chun, 2007; Olson \& Chun, 2002). The combination of the present results and the findings of Brady and Chun suggest that near-peripheral (perifoveal) vision is used to make a snapshot of the local context around the target.

It should be noted that Brockmole, Castelhano, and Henderson (2006) recently showed that the importance of the spatial proximity of context information to the target does not hold for natural scenes. By varying the global and local contexts in real-world scenes, they showed that targets are more likely to be associated to global content than to local content. Since this variation is most likely dependent on the types of stimuli and environments that are used, it will be essential to test the role of peripheral vision in more naturalistic real-life scenes.

The results of the second experiment indicate that information that is learned through peripheral vision remains in memory for a long time period. Ten days after participating in the first experiment, the subjects were tested on a contextual-cuing task that contained the same repeated trials (repeated-old) as those used in the first experiment. A highly significant effect between the repeated-new and the repeated-old trials was found in the first epoch, indicating that the configurations remained in memory during those 10 days. Importantly, this could not be the result of the implicit contextual learning that took place within the second experiment, since the new configurations (repeated-new) were also repeated, thus correcting for new learning effects. The interaction effect that was found between epoch and condition indicates that the difference between repeated-new and repeated-old trials was larger in the first epoch than in the second epoch. Although the long-term contextual-cuing effect was reflected in the first epoch, it can be speculated that it disappears in the second epoch because of learning of the repeated-new configurations during the second experiment, resulting in shorter response times in the second epoch. Indeed, repeated-new configurations remained new only during the first epoch. As a result of this learning effect, the difference between the repeated-old and repeated-new trials became smaller in the second epoch.

It should be noted that in the second experiment, the same subjects were tested as in the first experiment, so the subjects could have expected that repeated configurations would be used, even though they were not informed about the repetitions in the second experiment. However, none of the subjects indicated having noticed the repetitions, nor did they perform above chance level on the recognition task. This rules out the possibility that explicit memory processes were involved in the second experiment.

The distinction between peripheral and central vision has often been made when the retina and low-level cortical correlates of basic visual functions, such as contrast sensitivity or local speed discrimination (Anderson, Drasdo, \& Thompson, 1995; Castelo-Branco et al., 2006; Harris \& Fahle, 1996; Mendes et al., 2005; Silva et al., 2005), 
have been studied. However, a distinction between central and peripheral vision has rarely been made when higher cognitive functions, such as memory, have been studied. Only recently have a few researchers started to investigate the effect of eccentricity on specific higher order cognitive functions, such as object recognition. Levy, Hasson, Avidan, Hendler, and Malach (2001) suggested that some objects may be recognized better when perceived in central visual areas, whereas other objects are perceived better in peripheral vision. This distinction is reflected in visual cortical areas, in which different object categories have specific eccentricity biases (Levy et al., 2001; Levy, Hasson, Harel, \& Malach, 2004; Malach, Levy, \& Hasson, 2002). That is, objects that depend on analysis of fine detail, such as faces and words, are associated with central representations, whereas objects that involve integration of visual information over large retinal distances rely on peripheral visual areas. More recently, Liu and Ioannides (2006) found support for this idea by studying magnetoencephalographic responses to centrally and peripherally presented faces in a facial expression recognition task. They showed that the spatiotemporal dynamics and connectivity patterns were different for images presented to different parts of the visual field, thus supporting the idea that cortical visual processing depends very strongly on where the image appears in the visual field.

If object recognition is influenced by eccentricity, memory may also be affected by image position. Shoji and Skrandies (2006) showed that perceptual learning can take place in peripheral vision. Interestingly, Alexander, Packard, and Peterson (2002) showed that memory for the locations of objects (related to dorsal stream function) was better when the objects were presented in the peripheral visual field, as compared with central areas, suggesting a separation of peripheral memory processes and central memory processes. Further knowledge regarding the effect of where information appears in the visual field on memory is limited - in particular, how peripherally perceived information is processed into memory.

Although it was clearly shown that spatial context information that is perceived in the peripheral visual field can be learned, it remains unclear whether this process is also responsible for the contextual-cuing effect that has been found in studies in which subjects have been allowed to foveate the contextual information. Considering the nature of spatial context information, peripheral vision might dominate contextual learning, since it is impossible to foveate to large objects or spatial relations between objects that span several degrees in visual space. Future studies will have to determine whether contextual information can be learned by both central and peripheral vision, or whether it depends on a specialized system based on peripheral vision.

The present findings are also important for the discussion regarding the role of attention in implicit contextual learning. Although it is clear that memory can guide attention, the role of attention in implicit learning is still under debate. Recent studies have demonstrated that attention might not be necessary for implicit contextual learning (Jiang \& Leung, 2005; Tseng \& Li, 2004), although these studies refer to overt attention, since target and context information can be foveated. In our study, not even target stimuli were overtly attended by means of fixations (they had to be covertly searched for), which ensured that context could be stored only within the framework of peripheral representations. Therefore, the present study suggests that covert attention might play a facilitatory role in implicit contextual learning. By making only covert shifts of attention to the stimuli in the periphery, the subjects were still able to learn the spatial relations between the stimuli. Subsequently, covert attention could be guided by memory in order to locate the targets more quickly. These findings extend the results of previous studies showing that covert attention might play an important role in memory. For example, short-term priming of location or stimulus features has been found for covert attention (Maljkovic \& Nakayama, 1994, 1996). Furthermore, our findings extend previous knowledge on perceptual-priming tasks, in which learning is known to occur independently of awareness (Joyce \& Kutas, 2005; Meister et al., 2005; Wiggs \& Martin, 1994, 1998).

In conclusion, this study uncovered the fact that during implicit contextual learning, covert shifts of attention enable contextual information to be perceived and stored with peripheral vision. This peripherally perceived information can remain in memory for at least 10 days, and up to that time, it guides covert attention to the target location. It would be interesting if future research directly compared parallel processing within peripheral and central vision.

\section{AUTHOR NOTE}

This research was supported by a grant from the BIAL Foundation (No. 73/06) and the FCT (SFRH/BPD/22088/2005). Correspondence concerning this article should be addressed to M. van Asselen, IBILIFaculdade de Medicina, University of Coimbra, Azinhaga de Santa Comba-Celas, 3000-354 Coimbra, Portugal (e-mail: masselen@ibili .uc.pt).

\section{REFERENCES}

Alexander, G. M., Packard, M. G., \& Peterson, B. S. (2002). Sex and spatial position effects on object location memory following intentional learning of object identities. Neuropsychologia, 40, 1516-1522. doi:10.1016/S0028-3932(01)00215-9

Anderson, S. J., Drasdo, N., \& Thompson, C. M. (1995). Parvocellular neurons limit motion acuity in human peripheral vision. Proceedings of the Royal Society B, 261, 129-138. doi:10.1098/rspb.1995.0127

Brady, T. F., \& Chun, M. M. (2007). Spatial constraints on learning in visual search: Modeling contextual cuing. Journal of Experimental Psychology: Human Perception \& Performance, 33, 798-815. doi:10.1037/0096-1523.33.4.798

Brockmole, J. R., Castelhano, M. S., \& Henderson, J. M. (2006) Contextual cueing in naturalistic scenes: Global and local contexts. Journal of Experimental Psychology: Learning, Memory, \& Cognition, 32, 699-706. doi:10.1037/0278-7393.32.4.699

Castelo-Branco, M., Mendes, M., Silva, M. F., Januário, C., Machado, E., Pinto A., ET AL. (2006). Specific retinotopically based magnocellular impairment in a patient with medial visual dorsal stream damage. Neuropsychologia, 44, 238-253. doi:10.1016/ j.neuropsychologia.2005.05.005

Chun, M. M. (2000). Contextual cueing of visual attention. Trends in Cognitive Sciences, 4, 170-178. doi:10.1016/S1364-6613(00)01476-5

Chun, M. M., \& JiAng Y. (1998). Contextual cueing: Implicit learning and memory of visual context guides spatial attention. Cognitive Psychology, 36, 28-71.

Chun, M. M., \& JiAng, Y. (2003). Implicit, long-term spatial contextual 
memory. Journal of Experimental Psychology: Learning, Memory, \& Cognition, 29, 224-234. doi:10.1037/0278-7393.29.2.224

HARRIS, J. P., \& FAHLE, M. (1996). Differences between fovea and periphery in the detection and discrimination of spatial offsets. Vision Research, 36, 3469-3477. doi:10.1016/0042-6989(96)00076-4

JiANG, Y., \& LeUNG, A. W. (2005). Implicit learning of ignored visual context. Psychonomic Bulletin \& Review, 12, 100-106.

JIANG, Y., SoNG, J.-H., \& Rigas, A. (2005). High-capacity spatial contextual memory. Psychonomic Bulletin \& Review, 12, 524-529.

Joyce, C. A., \& Kutas, M. (2005). Event-related potential correlates of long-term memory for briefly presented faces. Journal of Cognitive Neuroscience, 17, 757-767. doi:10.1162/0898929053747603

Levy, I., Hasson, U., Avidan, G., Hendler, T., \& Malach, R. (2001). Center-periphery organization of human object areas. Nature Neuroscience, 4, 533-539. doi:10.1038/87490

Levy, I., Hasson, U., Harel, M., \& Malach, R. (2004). Functional analysis of the periphery effect in human building related areas. Human Brain Mapping, 22, 15-26. doi:10.1002/hbm.20010

LiU, L., \& IoANnides, A. A. (2006). Spatiotemporal dynamics and connectivity pattern differences between centrally and peripherally presented faces. NeuroImage, 31, 1726-1740. doi:10.1016/ j.neuroimage.2006.02.009

Malach, R. M., Levy, I., \& Hasson, U. (2002). The topography of high-order human object areas. Trends in Cognitive Sciences, 6, 176184. doi:10.1016/S1364-6613(02)01870-3

Maljkovic, V., \& Nakayama, K. (1994). Priming of pop-out: I. Role of features. Memory \& Cognition, 22, 657-672.

Maljkovic, V., \& Nakayama, K. (1996). Priming of pop-out: II. The role of position. Perception \& Psychophysics, 58, 977-991.

Meister, I. G., Weidemann, J., Foltys, H., Brand, H., Willmes, K., KRINGS, T., ET AL. (2005). The neural correlate of very-long-term picture priming. European Journal of Neuroscience, 21, 1101-1106. doi:10.1111/j.1460-9568.2005.03941.x
Mendes, M., Silva, F., Simões, L., Jorge, M., Saraiva, J., \& CasteloBRANCO, M. (2005). Visual magnocellular and structure from motion perceptual deficits in a neurodevelopmental model of dorsal stream function. Cognitive Brain Research, 25, 788-798. doi:10.1016/j .cogbrainres.2005.09.005

Olson, I. R., \& Chun M. M. (2002). Perceptual constraints on implicit learning of spatial context. Visual Cognition, 9, 273-302. doi:10.1080/ 13506280042000162

Peterson, M. S., \& Kramer A. F. (2001). Attentional guidance of the eyes by contextual information and abrupt onsets. Perception \& Psychophysics, 63, 1239-1249.

ShOJI, H., \& SKRANDIES, W. (2006). ERP topography and human perceptual learning in the peripheral visual field. International Journal of Psychophysiology, 61, 179-187.

Silva, F., Faria, P., Regateiro, F., Forjaz, V., Januário, C. Freire, A., \& Castelo-Branco, M. (2005). Independent patterns of damage within magno-, parvo- and koniocellular pathways in Parkinson's disease. Brain, 128, 2260-2271. doi:10.1093/brain/awh581

TsenG, Y. C., \& LI, C.-S. R. (2004). Oculomotor correlates of contextguided learning in visual search. Perception \& Psychophysics, 66, 1363-1378.

Wiggs, C. L., \& Martin, A. (1994). Aging and feature-specific priming of familiar and novel stimuli. Psychology \& Aging, 9, 578-588 doi:10.1037/0882-7974.9.4.578

Wiggs, C. L., \& Martin, A. (1998). Properties and mechanisms of perceptual priming. Current Opinion in Neurobiology, 8, 227-233. doi:10.1016/S0959-4388(98)80144-X

(Manuscript received June 18, 2008; revision accepted for publication July 26, 2008.) 